\title{
Impact of respiratory viral infections on cystic fibrosis
}

\section{Wat}

Postgrad Med J 2003;79:201-203

The life expectancy for patients with cystic fibrosis has improved remarkably over the last 20 years. Progressive deterioration of pulmonary function continues despite the aggressive use of antimicrobials. The absence of fever, neutrophilia, and systemic symptoms suggest that during pulmonary exacerbations other non-bacterial factors may have played a part. Some have suggested respiratory viruses as main suspects. So far, few data have illustrated the relationship of respiratory viruses and cystic fibrosis. By gaining further knowledge of this relationship, one may change future clinical practice and boost the survival of these patients.
Correspondence to: Dr Dennis Wat, University of Wales College of Medicine, Heath Park, Cardiff CF 14 4XW, UK; denniswat118@hotmail.com

Submitted

9 October 2002

Accepted 9 March 2003

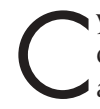
ystic fibrosis is an autosomal recessive condition with a prevalence of one in 2500. It is a multisystem disorder that can affect the respiratory, gastrointestinal, liver, skin, and genitourinary system.

It has been over 60 years since the first description of the disease and has been 10 years since the localisation of the genetic defect to the long arm of chromosome $7 .^{1}$ The gene product is the 1480 amino acid and the cystic fibrosis transmembrane conductance regulator, a protein that normally regulates and participates in the transport of electrolytes across epithelial cell membranes and possibly also across intracellular membranes. ${ }^{2}$

About 1000 mutations have been identified for this gene so far. The most common worldwide mutation is at the delta F508 position on chromosome 7 . It also accounts for around $80 \%$ of mutations in the white British population. Identification of this gene mutation allows antenatal detection and provides scope for genetic counselling.

Life expectancy for patients with cystic fibrosis has increased dramatically over the last 40 years from a median of two years to a projected median survival of over 40 years of age. ${ }^{3}$ The improved survival to date is mainly down to the improvement of cystic fibrosis management-that is, optimising nutritional status, chest physiotherapy, and specialist centre care. ${ }^{4}$ Despite all these, progressive obstructive lung disease remains the major cause of death in children with cystic fibrosis. Once colonisation with Pseudomonas aeruginosa is established in the airways, progressive deterioration in lung function is observed. Even with the introduction of antibiotic treatment, the effect of slowing down the pulmonary damage is still limited. There is little information to define when $P$ aeruginosa colonisation begins and the co-factors which precipitate in its adherence to the respiratory epithelium.
Airway injury after respiratory viral infections has been suggested to influence colonisation and progressive lung disease. ${ }^{5}$ Therefore by preventing and understanding respiratory viral infections in cystic fibrosis, it may delay the development of pulmonary dysfunction and reduce the risk of $P$ aeruginosa colonisation.

\section{VIRUSES IN CYSTIC FIBROSIS}

For many years, it has been the general opinion that respiratory viruses have been the cause of the "common cold" but are able to produce serious complications in infants, the elderly, and the immunocompromised. Studies of asthmatic children have demonstrated a significant increase in pulmonary symptoms at the time of respiratory viral infections. ${ }^{6}$ The association between respiratory viruses and asthma has long been well described. ${ }^{6-8}$

However, only a few prospective studies have reported the potential significance of respiratory viruses in cystic fibrosis. ${ }^{9-14}$ Some of these studies suggested that $40 \%$ of acute pulmonary exacerbations in cystic fibrosis are associated with respiratory viruses; these viruses also lead to pulmonary function abnormalities and disease progression. Armstrong et al showed that $52 \%$ of the infants with cystic fibrosis admitted to the hospital with respiratory symptoms were caused by respiratory viruses. ${ }^{15}$ Thirty five percent of these hospitalised infants acquired $P$ aeruginosa at follow up compared with $6 \%$ of those who were not hospitalised for respiratory symptoms. The author concluded that respiratory viral infections were associated with airway inflammatory changes, and admission to hospital was associated with early acquisition of $P$ aeruginosa.

This evidence is further supported by Petersen et al who found seven of 116 patients with cystic fibrosis developed the onset of chronic $P$ aeruginosa colonisation after viral infection. ${ }^{10}$ Yet, these studies may have underestimated the importance of respiratory viruses in cystic fibrosis based on four reasons:

(1) The low detection rates by tissue culture and immunofluorescence techniques were disappointing. In most published studies all of the infections were diagnosed by serology. ${ }^{10} 1113$

(2) DNA based methods can identify up to 10 times more positive samples for some viruses. ${ }^{16}$

(3) Most studies have been hampered by a limited use of control subjects, the lack of use of objective indicators, such as pulmonary function tests and chest radiographs to define pulmonary exacerbations.

(4) There is great difficulty in isolating viruses from tissue culture from cystic fibrosis patients, ${ }^{10}{ }^{11}$ which may be related to the thick mucoid secretion from their airways. This may further hinder detection by immunofluorescence. 


\section{VIRUS DETECTION METHODS}

The conventional methods of viral detection such as serology, immunofluorescence, and culture have low sensitivities. Rhinoviruses and coronaviruses account for $75 \%$ of upper respiratory infections. Since there are more than 100 serotypes of rhinoviruses, accurate diagnosis by such methods became impossible and unreliable. These conventional methods are also time consuming, they may take up to a week to yield positive viral cultures. Therefore this also makes them impractical in the clinical setting where accuracy and rapid detection are paramount.

Molecular based techniques such as polymerase chain reaction is five times more sensitive than tissue culture techniques. ${ }^{17}$ Apart from accuracy, viral detection can also be made in a matter of hours rather than days. In addition, it can provide quantitative measure for viral load. It is particularly helpful in the case of rhinoviruses, which are notorious for their culture fastidiousness because of the great number of serotypes. Whereas in the past, there was no standard successful detection method for coronaviruses, they can now be easily detected by molecular techniques

\section{INTERACTION BETWEEN BACTERIA AND RESPIRATORY VIRUSES}

Several studies have suggested that respiratory viral infections predispose to secondary bacterial infections and colonisation. $^{15} 18$

Collinson et al showed that 10 of the 12 cystic fibrosis children with positive sputum bacterial culture also had positive viral detection from nasopharyngeal specimens. ${ }^{18}$ The viruses were detected by the polymerase chain reaction. Yet the exact relationship between respiratory viruses and bacterial colonisation in cystic fibrosis individuals is unclear. Some have suggested that respiratory viral infections cause injury to respiratory epithelium, which in turn leads to increased adherence of pharyngeal cells to bacterial infections such as Staphylococcus aureus, Haemophilus influenzae, Streptococcus pneumonia, and $P$ aeruginosa. ${ }^{19} 20$

When Armstrong et al examined the bronchiolar lavage fluid from cystic fibrosis patients with clear evidence of viral infection, they found that there were increased interleukin- 8 levels and elastase activity compared with control subjects. ${ }^{21}$ This increased elastase activity was further shown to increase pseudomonas adherence to tracheal cells. ${ }^{22}$

Przyklenk et al recruited 75 cystic fibrosis patients with a mean age of 15 years and showed that $93 \%$ of them with positive serological conversion to respiratory viruses had a threefold increase in bacterial colony forming units per millimetre of sputum. ${ }^{23}$ Seventy eight percent of them also had evidence of pulmonary deterioration. The authors therefore concluded that there was a strong "microbial synergism" between the bacterial flora of the sputum and infection with respiratory viruses.

\section{TREATMENT FOR RESPIRATORY VIRAL INFECTION}

Vaccines offer the possibility of decreasing the severity and complications of viral respiratory disease. However, the success of vaccination has been limited by significant variation within the major virus types causing disease.

There are 102 serotypes of rhinovirus and no effective vaccine has been introduced. Influenza viruses exhibit antigenic shift and drift. The development of a safe and effective vaccine against respiratory syncytial virus (RSV) remains an important challenge because of the major setback in the 1960s, when formalin-inactivated whole RSV vaccine caused $80 \%$ of vaccinees to become hospitalised, compared with 5\% of controls. ${ }^{24}$ Two deaths were observed among RSV vaccinees as a result of disease augmentation. Current major research work has been focusing on prophylaxis using a humanised mouse monoclonal antibody, palivizumab. It has been proved to be safe and effective and gained approval by the United States Food and Drug Administration in 1998. However, it requires monthly administration during the RSV season and its benefits in cystic fibrosis are currently being evaluated.

In man, natural infection with RSV causes mild respiratory symptoms and is generally self limiting. But the natural immunity against it is short lived and reinfections with serologically similar strains are frequent throughout life. Anti-RSV vaccine would therefore have to induce a "better-than-life" immunity and offers good protection for the duration of the winter season.

The other approach to deal with respiratory viral infections is by the use of specific antiviral drugs, but this requires rapid accurate diagnosis, where molecular viral detection methods are once again so important.

Specific viral agents do exist for influenza viruses such as amantidine, rimantidine, and more recently developed neuraminidase inhibitors such as zanamivir and oseltamivir. Their use, however, is restricted to adults. Wright et al attempted to evaluate the safety of amantidine in children with cystic fibrosis who had respiratory viral infections. ${ }^{25}$ Unfortunately, influenza virus infection did not reach epidemic proportions during the study period, therefore the effectiveness of amantidine could not be fully assessed.

Nebulised ribavarin is licensed for the use of hospitalised infants and children in the first three days of RSV bronchiolitis. It is a nucleotide analogue, active against RSV in vitro; however, it is expensive and of no proved clinical benefit.

As mentioned previously, rhinovirus is a major cause of "common colds" in young children. The development of drug therapy against rhinovirus poses the greatest challenge. Picovir is a capsid binding/canyon inhibitor which blocks the binding of rhinoviruses to the host cell receptor. It is very potent, but its clinical usefulness is often limited by serotype specificity and the rapid emergence of resistance.

\section{CONCLUSION}

Respiratory viruses have been implicated in pulmonary exacerbations of cystic fibrosis and cause long term pulmonary damage in these patients. However, the current data available are by no means comprehensive and further evaluations of the role of respiratory viruses in the cystic fibrosis populations are required.

With prior knowledge of the impact of respiratory viruses in the exacerbation of cystic fibrosis, routine screening of respiratory viruses during pulmonary exacerbations should be implemented in clinical practice. This assessment should include obtaining specimens from the respiratory tract and using molecular viral detection methods to reach a rapid diagnosis. The identification of respiratory viruses may subsequently alter our clinical practice. The patient can be isolated appropriately, and this can also remove the undesirable side effects of antimicrobials, ${ }^{26}$ especially if they are being prescribed unnecessarily.

If respiratory viruses do induce reactive airway obstruction, the current practice of prescribing bronchodilator therapy would be appropriate. If, on the other hand, the principal mechanism of viral-induced injury is found to be through synergistic interaction with bacteria, the current practice of antimicrobial therapy would remain appropriate.

The role of antiviral therapy will need further evaluation with well designed randomised control trials. Whereas for vaccination, further understanding of the complex viral immune response will aid us to develop safe and multivalent vaccines which offer good protection against a spectrum of respiratory viruses.

Without a doubt, bacterial pathogens are still main causes of pulmonary exacerbations in cystic fibrosis and aggressive antimicrobial therapy is one of the important reasons for the dramatic increase in life expectancy over the last few 
decades. ${ }^{27} 28$ In fact prophylactic antibiotics are advocated by some groups, started at the time of diagnosis. But recent studies also suggested that continuous prophylactic treatment with antistaphylococcus may increase the risk of early colonisation with pseudomonas. One would wonder if antimicrobials are being over prescribed. With gene therapy still undergoing further research with regards to its validity and specificity, gaining further understanding in the molecular mechanism of virus induced respiratory exacerbations may allow the development of new therapeutic techniques. At the same time we must bear in mind the disappointing results of antivirals. The development of new strategies will be exciting and this may further prolong the lifespan of patients with cystic fibrosis and more importantly improve their quality of life.

\section{REFERENCES}

1 Riodren JR, Rommens JM, Kerem B, et al. Identification of the cystic fibrosis gene: cloning and characteristion of complementary DNA. Science 1989:245: 1066-73

2 Barasch J, Al-Awagti Q. Defective acidification of the biosynthetic pathway in cystic fibrosis. J Cell Sci 1993;17(suppl 1):229-33.

3 Elborn JS, Shale DJ, Britton JR. Cystic fibrosis: current survival and Elborn JS, Shale DJ, Britton JR. Cystic fibrosis: current survival and
population estimates to the year 2000. Thorax 1991:46:881-5.

4 Mahadeva R, Webb K, Westerbeak RC, et al. Clinical outcome in relation to care in centres specialising in cystic fibrosis-a cross sectional study. BM 1998;316:1771-5.

5 Accurso FJ, Sokol RJ, Hammond KB, et al. Early respiratory course in infants with cystic fibrosis: relevance to newborn screening. Pediatr Pulmonol 1991; 7:42-5.

6 Johnson SL, Pattemore PK, Sanderson G, et al. Community study of the role of viral infections in exacerbations of asthma in 9-11 year old children. BM 1995;310:1225-8.

7 Gern JE, Williams WB. Association of rhinovirus infections with asthma. Clin Microbiol Rev 1999;12:9-18.

8 Freymouth F, Vabret A, Brouard J, et al. Detection of viral Chlamydia pneumoniae and mycoplasma pneumoniae infections in exacerbations of asthma. J Clin Virol 1999;13:131-9.

9 Wright PF, Khaw KT, Oxman MN, et al. Evaluation of the safety of amantadine-HC 1 and the role of respiratory viral infections in children with cystic fibrosis. J Infect Dis 1976; 134:144-9.

10 Petersen NT, Hoiby N, Mordhorst CH, et al. Respiratory infections in cystic fibrosis patients caused by virus, chlamydia and mycoplasma; possible synergism with Pseudomonas aeruginosa. Acta Paediatr Scand 1981;70:628-8.

11 Wang EE, Prober CG, Manson B, et al. Association of respiratory viral infections with pulmonary deterioration in patients with cystic fibrosis. $N$ Engl J Med 1984;311:1653-8.
12 Abman SH, Ogle JW, Butlet SN, et al. Role of respiratory syncytial virus in early hospitalisation for respiratory distress of young infants with cystic fibrosis. J Pediatr 1988;113:826-30.

13 Ramsey BM, Gore EJ, Smith AL, et al. The effect of respiratory viral infections on patients with cystic fibrosis. Am J Dis Child 1989;143:662-8

14 Smyth AR, Smyth RL, Tony CYW, et al. Effect of respiratory viral infections including rhinovirus in clinical status in cystic fibrosis. Arch Dis Child 1995;73:1 17-20.

15 Armstrong D, Grimwood K, Carlin JB, et al. Severe viral respiratory infections in infants with cystic fibrosis. Pediatr Pulmonol 1998:26:371-9.

16 Freymouth F, Vabret A, Galateau-Salle F, et al. Detection of respiratory syncytial virus, parainfluenza virus, adenovirus and rhinovirus sequences in respiratory tract of infants y polymerase chain reaction and hybridisation. Clinical Diagnosis Virology 1997;8:31-40.

17 Ireland DC, Kent J, Nicholson KG. Improved detection of rhinovirus in nasal and throat swabs by semi-nested RT-PCR. J Med Virol 1993:40:96-101.

18 Collinson J, Nicholson KG, Cancio E, et al. Effects of respiratory tract infections in patients with cystic fibrosis. Thorax 1996;51:1115-22.

19 Ramirez-Randa CH, Fuxench-Lopez Z, Nevarez M, et al. Increased pharyngeal bacterial colonisation during viral illnesses. Arch Intern Med $1981 ; 114: 599-603$.

20 Smith CB, Golden C, Klauber MR, et al. Interaction between viruses and bacteria in patients with chronic bronchitis. $J$ Infect Dis 1976;134:552-61.

21 Armstrong DS, Grimwood K, Carlin JB, et al. Lower airway inflammation in infants and young children with cystic fibrosis. Am J Respir Crit Care Med 1997:156(4 pt 1):1197-204.

22 Niederman MS, Merrill WW, Ferrenti RD, et al. Nutritional status and bacterial binding in the lower respiratory tract in patients with chronic tracheostomy. Ann Intern Med 1984;100:795-800

23 Przyklenk B, Bavernfeind A, Bertele RM, et al. Viral infections of the respiratory tract in patients with cystic fibrosis. Serodiagn Immunother Infect Dis 1988:2:217-25.

$24 \mathrm{Kim}$ HW, Canchola JG, Brandt CD, et al. Respiratory syncytial viral disease in infants despite prior administration of antigen inactivated vaccine. Am J Epidemiol 1969;89:422-34.

25 Wright PF, Khaw KT, Oxman MN, et al. Evaluation of the safety of amantadine-HC 1 and the role of respiratory viral infections in children with cystic fibrosis. J Infect Dis 1976;134:144-9.

26 Ratjen R. Changes in strategies for optimal antibacterial therapy in cystic fibrosis. Int J Antimicrob Agents 2001;17:93-6.

27 Mukhopadhyay S, Singh M, Cater Jl, et al. Nebulised anti-pseudomonal antibiotic therapy in cystic fibrosis: a meta-analysis of benefits and risks. Thorax 1996:551:361-8.

28 Ramsay BW, Pepe MS, Quan JM, et al. Intermittent administration of inhaled tobramycin in patients with cystic fibrosis. N Engl J Med 1999;340:23-30 